\title{
Gravidade da cardiopatia congênita e nível de redução da variabilidade da frequência cardíaca
}

\author{
Severity of congenital heart disease and level \\ of reduction in heart rate variability
}

Moacir Fernandes de Godoy',2, Gabriela Nascimento Marques', João Maeda', Bruna Bento Madalozzo', Larissa Helena Marques Carrai', Giovana Davi Lorente', Karolyne Barroca Sanches ${ }^{3}$, Carlos Marcelo Pastre ${ }^{2,4}$, Luiz Carlos Marques Vanderlei ${ }^{2,4}$

\section{Resumo}

Introdução: A variabilidade da frequência cardíaca (VFC) tem sido utilizada como medida do balanço autonômico, ou seja, da relação entre o sistema simpático e o parassimpático, sendo, indiretamente, um indicativo do nível homeostático do indivíduo. Há relatos de redução da VFC em cardiopatias congênitas, mas não há estudos mais sistematizados buscando correlação entre a gravidade estrutural e funcional da cardiopatia e o grau de redução da variabilidade. Objetivo: Conduzir um estudo piloto em um grupo de cardiopatas congênitos buscando indícios de menor VFC correlacionada à pior condição estrutural ou funcional da cardiopatia em questão. Método: Foram avaliados 17 pacientes, sendo 11 portadores de cardiopatias congênitas consideradas de baixo impacto estrutural e seis com cardiopatias cianogênicas graves. Foi feita a captação das séries temporais por meio do equipamento Polar RS800 ${ }^{\circledR}$ em situação de repouso, na posição supina, no período da manhã, selecionando-se 1 mil intervalos RR consecutivos para análise com o software Kubios HRV Analysis, de variáveis nos domínios do tempo (SDNN, RMSSD), no domínio da frequência (LFms² ${ }^{2}$ HFms²) e não lineares (SD1, SD2 e Entropia de Shannon). Utilizou-se um grupo de 21 crianças normais como controle. Resultados: Foi possível detectar redução da VFC nos três domínios estudados, com valor $p$ limítrofe para significância. E para a Entropia de Shannon, detectou-se diferença estatística $(p=0,0020)$, indicando que o grupo com maior comprometimento estrutural ou funcional apresenta pior balanço homeostático. Conclusão: Pacientes portadores de cardiopatia congênita com importante comprometimento estrutural e/ou funcional apresentam redução da VFC, traduzindo desbalanço homeostático.

Palavras-chave: cardiopatias congênitas; frequência cardíaca; homeostase.

\section{Abstract}

Introduction: The Heart Rate Variability (HRV) has been used as a measure of autonomic balance, or the relationship between the sympathetic and the parasympathetic system, and is indirectly indicative of the homeostatic level of an individual. There are reports also of reduced HRV in congenital heart diseases but no systematic studies searching for a correlation between the severity of functional and/or structural heart disease and the degree of reduction of HRV. Objective: To conduct a preliminary study in a group of congenital heart diseases searching for signs of lower HRV correlated to a more severe structural or functional heart disease. Method: We evaluated 17 patients, 11 patients with congenital heart disease considered of low-impact structural/functional and six with severe cyanogenic disease. The temporal series were captured with the Polar RS800 ${ }^{\circledR}$ device in a supine position, during the morning period, selecting 1thousand consecutive RR intervals for analysis with the software Kubios HRV Analysis of variables in the time domain (SDNN, RMSSD) in the frequency domain (LFms², HFMms²) and nonlinear domain (SD1, SD2 and Shannon Entropy). As a control group were included 21 healthy children. Results: It was possible to detect reduced HRV in the three domains studied with $p$ value of borderline significance and for the Shannon Entropy there was a significant statistical difference $(p=0.0020)$, indicating that the group with greater structural/functional impairment

\footnotetext{
Trabalho realizado na Faculdade de Medicina de São José do Rio Preto (FAMERP) - São José do Rio Preto (SP), Brasil.

${ }^{1}$ Faculdade de Medicina de São José do Rio Preto (FAMERP) - São José do Rio Preto (SP), Brasil.

${ }^{2}$ Núcleo Transdisciplinar para Estudo do Caos e da Complexidade (NUTECC) - São José do Rio Preto (SP), Brasil.

${ }^{3}$ Serviço de Cirurgia Cardíaca Pediátrica do Hospital de Base de São José do Rio Preto (FUNFARME) - São José do Rio Preto (SP), Brasil.

${ }^{4}$ Faculdade de Ciências e Tecnologia de Presidente Prudente (FCT/UNESP) - Presidente Prudente (SP), Brasil.

Endereço para correspondência: Moacir Fernandes de Godoy - Rua Garabed Karabashian, 570 - Parque Residencial Comendador Mancor Daud -

CEP: 15070-600 - São José do Rio Preto (SP), Brasil - E-mail: mf60204@gmail.com

Fonte de financiamento: nenhuma.

Conflito de interesse: nada a declarar.
} 
has the lowest variability. Conclusion: Patients with congenital heart disease with significant functional and structural impairment have reduced HRV reflecting homeostatic imbalance.

Keywords: heart defects, congenital; heart rate; homeostasis.

Recebido em: 20/08/2011

Revisado em: 19/12/2011

Aprovado em: 22/02/2012

\section{Introdução}

O sistema nervoso autônomo (SNA) desempenha papel importante na regulação dos processos fisiológicos do organismo humano tanto em condições normais quanto patológicas. Dentre as técnicas utilizadas para sua avaliação, a variabilidade da frequência cardíaca (VFC) tem emergido como uma medida simples e não invasiva dos impulsos autonômicos. A VFC descreve as oscilações no intervalo entre batimentos cardíacos consecutivos (intervalos RR), assim como oscilações entre frequências cardíacas instantâneas consecutivas ${ }^{1}$.

A VFC constitui um potente e independente indicador de mortalidade cardiovascular. Atualmente, com o auxilio da tecnologia digital, tem havido aumento crescente na utilização desse recurso, realizando-se análise sob três focos específicos, chamados domínios - do tempo e da frequência (métodos lineares) e do caos (método não linear) -, tanto em condições normais quanto patológicas.

A perda ou a redução da VFC reflete indiretamente a redução do comportamento homeostático. Em comparação entre neonatos pré-termo e recém-nascidos eutróficos e sem eventos perinatais, observou-se diferença significante para a variabilidade tanto nos domínios do tempo e da frequência quanto no domínio do caos $^{2}$. Além disso, existem evidências que relatam alterações da VFC em crianças obesas quando comparadas às eutróficas ${ }^{3}$.

Alguns estudos mostram que nas cardiopatias congênitas a VFC é reduzida dependendo da limitação funcional apresentada pela criança, de forma que os índices apresentados tornam-se marcadores importantes de estados clínicos ${ }^{4}$. Em crianças com comunicação interatrial (CIA) podem ocorrer arritmias, cuja expressão depende do retorno venoso pulmonar ou de um controle normal do sistema nervoso autonômico. Nelas, foi demonstrada uma baixa VFC, correspondente à arritmia, em íntima relação com a cardiopatia. Após a correção da CIA, as crianças apresentaram melhora do quadro clínico, concomitante com aumento da VFC5

Em comparação feita entre crianças com comunicação interventricular (CIV) e CIA, a resposta respiratória vagal (parassimpático) na CIA é menor, sendo que ambas apresentam menor VFC do que o normal. Essa menor resposta vagal da CIA deve-se ao aumento do volume e consequente estiramento mecânico do átrio direito ${ }^{6}$.

A literatura é escassa em termos de estudo da VFC em portadores de cardiopatia congênita, principalmente em nosso meio. Sabe-se que a redução da variabilidade cardíaca está relacionada à ocorrência de eventos deletérios, daí a relevância da realização de um estudo com esse enfoque.

Assim, o objetivo foi descrever o comportamento da VFC em indivíduos cardiopatas congênitos, bem como buscar possíveis associações entre a redução da VFC e maior comprometimento estrutural/ funcional pela cardiopatia.

\section{Método}

Foram avaliados 17 pacientes, sendo 11 portadores de cardiopatias congênitas consideradas de baixo impacto estrutural (estenose valvar aórtica: quatro; estenose valvar pulmonar: três; CIV: dois; defeito do septo atrioventricular: um; bradicardia: um) e seis com cardiopatias cianogênicas graves (atresia tricúspide: dois; truncus arteriosus, atresia pulmonar, dupla via de entrada de ventrículo esquerdo e transposição completa dos grandes vasos da base: um cada). Foi feita a captação das séries temporais por meio do equipamento Polar RS800 ${ }^{\circledR}$ em situação de repouso na posição supina, sempre no período entre 7 e 12 horas. Esse equipamento detecta as ondas RR do eletrocardiograma (ECG) com resolução temporal de um milissegundo (ms), já estando devidamente validado ${ }^{7-8}$. As séries de intervalos RR foram filtradas de forma automática e visual. Somente os registros com mais de $95 \%$ de batimentos de origem sinusal foram qualificados, selecionando-se 1 mil intervalos RR consecutivos para análise com o software Kubios HRV Analysis, de variáveis nos domínios do tempo (SDNN, RMSSD), no domínio da frequência $\left(\mathrm{LFms}^{2}, \mathrm{HFms}^{2}\right)$ e não lineares (SD1, SD2 e Entropia de Shannon). Utilizou-se um grupo de 21 crianças normais como controle. O presente estudo foi aprovado pelo Comitê de Ética em Pesquisa (CEP) da Faculdade de Medicina de São José do Rio Preto, São Paulo, sob o parecer $348 / 2011$.

\section{Análise estatística}

As variáveis quantitativas com distribuição gaussiana foram comparadas por análise de variância, e as comparações pós-teste feitas em relação ao controle normal pelo teste de comparação de Dunnet. Para representação gráfica utilizaram-se gráficos tipo Box-Plot [valor mínimo; médiatintervalo de confiança 95\%; valor máximo]. Foi admitido erro alfa de $5 \%$, sendo considerados significantes valores $p \leq 0,05$. 


\section{Resultados}

Foi possível detectar redução da VFC nos três domínios estudados com valor p limítrofe para significância, indicando que o grupo com maior comprometimento estrutural apresentava a menor variabilidade, ou seja, menor balanço homeostático em comparação ao controle normal. Para a Entropia de Shannon, que é uma variável do domínio não linear, foi possível detectar diferença estatisticamente significante $(\mathrm{p}=0,0020)$. Valores mais altos de Entropia de Shannon, nesse caso, indicam maior determinismo, ou seja, comportamento mais linear e, portanto, afastamento do estado homeostático. A Figura 1 destaca os gráficos Box-Plot de algumas das variáveis estudadas, e a Tabela 1 mostra o conjunto dos resultados obtidos no grupo de cardiopatias de baixa repercussão funcional (G1), no grupo de alta repercussão (G2) e no de crianças normais e os respectivos valores p obtidos na comparação estatística.

Com base neste estudo preliminar, o cálculo de tamanho amostral adequado indicou que com apenas 16 casos em cada grupo já é possível detectar diferenças estatísticas em todos os domínios, com poder superior a $80 \%$.

\section{Discussão}

O fato de ter sido detectada uma nítida tendência para comportamento diferenciado em termos de VFC em cardiopatias congênitas com alto impacto estrutural e funcional confirma a hipótese de que há nesse grupo um maior comprometimento da homeostase e uma possível explicação para

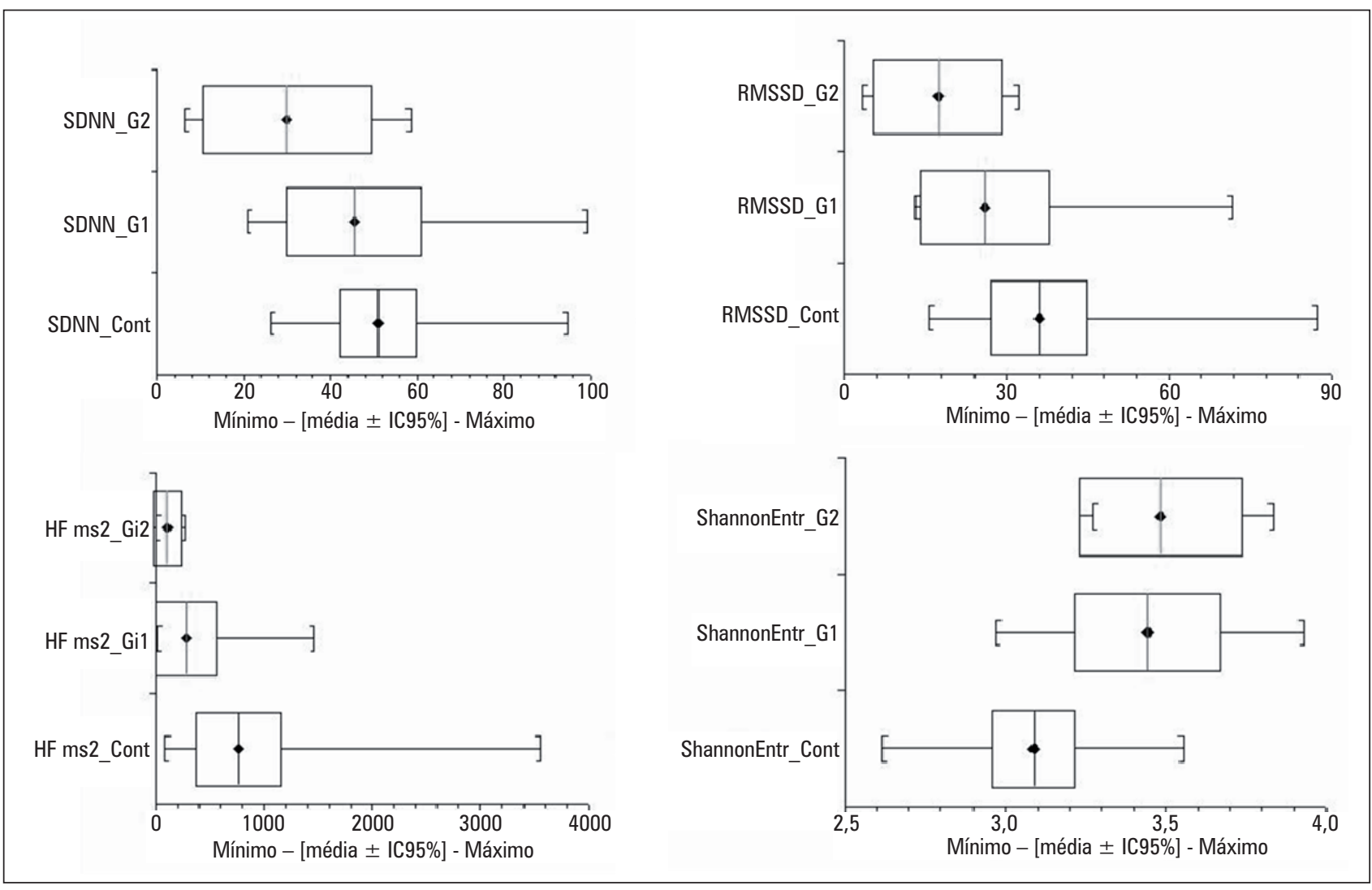

Figura 1: Gráficos Box-Plot das variáveis SDNN, RMSSD, HFms² e Entropia de Shannon, mostrando evidente redução da variabilidade da frequência cardíaca pelo componente parassimpático e aumento da Entropia de Shannon, indicando perda do comportamento não linear normal

Tabela 1: Valores das variáveis analisadas no grupo controle, grupo de cardiopatias com baixo impacto estrutural e funcional e grupo de cardiopatias congênitas com alto impacto estrutural e funcional

\begin{tabular}{lcccc}
\hline Variáveis & Controle & $\mathrm{G} 1$ & $\mathrm{G} 2$ & $\mathrm{p}$ \\
\hline Idade (anos) & $9,28 \pm 1,61$ & $11,00 \pm 9,55$ & $13,83 \pm 8,56$ & 0,2802 \\
SDNN (ms) & $51,00 \pm 18,97$ & $45,48 \pm 23,03$ & $29,88 \pm 18,59$ & 0,0912 \\
RMSSD (ms) & $36,03 \pm 19,52$ & $25,92 \pm 17,76$ & $17,36 \pm 11,41$ & 0,0673 \\
LFms $^{2}$ & $884,23 \pm 682,92$ & $678,45 \pm 750,18$ & $313,67 \pm 378,04$ & 0,1884 \\
HFms $^{2}$ & $762,04 \pm 862,41$ & $279,45 \pm 414,76$ & $108,67 \pm 120,57$ & 0,0614 \\
SD1 (ms) & $25,49 \pm 13,80$ & $18,34 \pm 12,55$ & $12,30 \pm 8,08$ & 0,0674 \\
SD2 (ms) & $67,17 \pm 23,85$ & $61,42 \pm 30,61$ & $40,38 \pm 25,17$ & 0,1010 \\
Entr.Shannon (bits) & $3,087 \pm 0,280$ & $3,441 \pm 0,339$ & $3,482 \pm 0,239$ & 0,0020 \\
\hline
\end{tabular}

G1: grupo de cardiopatias com baixo impacto estrutural e funcional; G2: grupo de cardiopatias congênitas com alto impacto estrutural e funcional; ms: milissegundo 
a maior probabilidade de eventos adversos, uma vez que a redução da VFC se associa a uma pior evolução, em diversas condições mórbidas.

É interessante ressaltar que Massin et al. ${ }^{4}$, já em 1998, procuraram detectar associação entre alterações da VFC e indicadores clínicos e hemodinâmicos em crianças portadoras de cardiopatia congênita. Para tanto, avaliaram 258 crianças com 11 estados diagnósticos pela VFC, sendo cinco variáveis no domínio do tempo (SDNN, SDNNi, SDANNi, rMSSD e pNN50) e três no da frequência (LF, HF e LF/HF), mas não houve a preocupação em separação dos casos por gravidade estrutural nem por condição operatória. Concluíram, porém, que o grau de comprometimento da VFC estava relacionado à gravidade da doença em termos da classe funcional pela New York Heart Association (NYHA), ou seja, quanto pior a classe funcional do paciente, menor a VFC. No presente estudo, todos os pacientes estavam compensados ou no máximo em classe funcional II da NYHA e, mesmo assim, foi detectada diferença entre os grupos, indicando que cardiopatas graves, mesmo clinicamente compensados, apresentam comprometimento da homeostase. Comentam também sobre o possível efeito protetor de drogas concomitantes como, por exemplo, digitálicos e inibidores de enzima de conversão de angiotensina, mas naquela casuística de 258 casos isso não foi relevante. Não houve a preocupação neste nosso estudo piloto, principalmente por ser a casuística ainda pequena, em detectar alterações da VFC relacionada com o uso ou não de medicamentos, todavia esses achados de Massin et al. ${ }^{4}$ parecem descartar essa necessidade. Os autores também não encontraram correlação entre o distúrbio hemodinâmico da cardiopatia (sobrecarga de volume ou pressão) e alteração da VFC, corroborando nossos achados de que é o distúrbio homeostático global o verdadeiro responsável pelo pior balanço autonômico.

As variáveis estudadas indicaram de forma persistente sempre uma tendência para redução da VFC à custa principalmente do componente parassimpático (menor RMSSD, menor HFms ${ }^{2}$, menor SD1 no gráfico de Poincaré) com manutenção do componente simpático
(LFms ${ }^{2}$ e SD2). A elevação significante no valor da Entropia de Shannon indica aqui perda da complexidade da VFC, o que também se coaduna com a maior gravidade do grupo G2.

A quase totalidade dos estudos de VFC em cardiopatias congênitas se limita à utilização de variáveis nos domínios do tempo e da frequência, sendo o uso de variáveis não lineares a exceção. Nosso estudo, ao constatar que a Entropia de Shannon foi um sensível marcador da maior gravidade estrutural e funcional da cardiopatia congênita, abre portas para pesquisas mais focadas nesse campo.

Apesar de em números absolutos a idade do grupo G2 ser um pouco maior que a do grupo controle, não houve diferença estatisticamente significante entre os grupos, nem mesmo limítrofe $(p=0,2802)$ e, além disso, é sabido que a VFC aumenta da criança em relação ao jovem adulto. Portanto, se houvesse algum viés nos resultados, favoreceria o Grupo G2, aumentando a VFC e não reduzindo, como foi o caso. Vale, por fim, ressaltar que realizou-se aqui apenas um estudo preliminar e, mesmo com pequena casuística, já foi possível essa constatação. Com base nos dados, está sendo realizada uma extensão da casuística, esperando-se, em breve, conclusões mais definitivas.

Pacientes portadores de cardiopatia congênita com importante comprometimento estrutural e funcional apresentam redução da VFC, traduzindo desbalanço homeostático, o que pode explicar a evolução clínica com maior probabilidade de eventos adversos desses casos. Davos et al. ${ }^{9}$ já demonstraram, em pacientes operados de Tetralogia de Fallot, que a alteração da VFC se mantém mesmo após vários anos decorridos da operação, e, esse fato, poderia estar relacionado a marcadores de taquicardia ventricular sustentada e morte súbita, sugerindo mecanismos fisiopatológicos comuns

Estudos mais aprofundados poderão ajudar a esclarecer as relações de causa e efeito desse achado.

\section{Referências}

1. Vanderlei LCM, Pastre CM, Hoshi RA, Carvalho TD, Godoy MF. Noções básicas de variabilidade da frequência cardíaca e sua aplicabilidade clínica. Rev Bras Cir Cardiovasc. 2009;24(2):205-17.

2. Selig FA, Tonolli ER, Silva EVCM, Godoy MF. Variabilidade da frequência cardíaca em neonatos prematuros e de termo. Arq Bras Cardiol. 2011;96(6):443-9

3. Vanderlei LC, Pastre CM, Freitas Júnior IF, Godoy MF. Analysis of cardiac autonomic modulation in obese and eutrophic children. Clinics. 2010;65(8):789-92

4. Massin M, von Bernuth G. Clinical and haemodynamic correlates of heart rate variability in children with congenital heart disease. Eur J Pediatr. 1998;157(12):967-71.

5. Finley JP, Nugent ST, Hellenbrand W, Craig M, Gillis DA. Sinus arrhythmia in children with atrial septal defect: an analysis of heart rate variability before and after surgical repair. Br Heart J. 1989;61(3):280-4.
6. Hata T, Mano S, Kusuki M, Matsuura H, Miyata M, Yamazaki $\mathrm{T}$, et al. Difference in autonomic nervous control between ventricular septal defect and atrial septal defect based on heart rate variability. Pacing Clin Electrophysiol. 2007;30(Suppl 1): S212-4.

7. Vanderlei LC, Silva RA, Pastre CM, Azevedo FM, Godoy MF. Comparison of the Polar S810i monitor and the ECG for the analysis of heart rate variability in the time and frequency domains. Braz J Med Biol Res. 2008;41(10) 854-9.

8. Gamelin FX, Berthoin S, Bosquet L. Validity of polar S810 heart rate monitor to measure R-R intervals at rest. Med Sci Sports Exerc. 2006;38: 887-93.

9. Davos CH, Davlouros PA, Wensel R, Francis D, Davies LC, Kilner PJ, et al. Global impairment of cardiac autonomic nervous activity late after repair of tetralogy of Fallot. Circulation. 2002;106(12 Suppl 1): 169-75. 